\title{
X-Chromosome Insufficiency Alters Receptive Fields across the Human Early Visual Cortex
}

\author{
Tamar Green, ${ }^{1}$ Hadi Hosseini, ${ }^{1}$ Aaron Piccirilli, ${ }^{1}$ Alexandra Ishak, ${ }^{1}$ @ Kalanit Grill-Spector, ${ }^{2,3,4 *}$ \\ and (D)Allan L. Reiss ${ }^{1,2,4,5 *}$ \\ ${ }^{1}$ Division of Interdisciplinary Brain Sciences, Department of Psychiatry and Behavioral Sciences, Stanford University, Stanford, California 94305 , \\ ${ }^{2}$ Neurosciences Program, Stanford University School of Medicine, Stanford, California 94305, ${ }^{3}$ Psychology Department, ${ }^{4}$ Stanford Neurosciences Institute, \\ and ${ }^{5}$ Departments of Radiology and Pediatrics, Stanford University, Stanford, California 94305
}

Here, we investigated processing by receptive fields, a fundamental property of neurons in the visual system, using fMRI and population receptive field (pRF) mapping in 20 human females with monosomic Turner syndrome (TS) (mean age, $10.3 \pm 2.0$ years) versus 22 ageand sex-matched controls (mean age, $10.4 \pm 1.9$ years). TS, caused by X-chromosome haploinsufficiency in females, is associated with well-recognized effects on visuospatial processing, parieto-occipital cortical anatomy, and parietal lobe function. However, it is unknown whether these effects are related to altered brain structure and function in early visual areas (V1-V3) versus downstream parietal cortical regions. Results show that girls with TS have the following: (1) smaller volume of V1-V3, (2) lower average pRF eccentricity in early visual areas, and (3) sparser pRF coverage in the periphery of the visual field. Further, we examined whether the lower volume of early visual areas, defined using retinotopic mapping, in TS is due to smaller surface area or thinner cortex. Results show that girls with TS had a general reduction in surface area relative to controls in bilateral V1 and V2. Our data suggest the possibility that the smaller cortical surface area of early visual areas in girls with TS may be associated with a lower number of neurons, which in turn, leads to lesser coverage of the peripheral visual field compared to controls. These results indicate that X-chromosome haploinsufficiency associated with TS affects the functional neuroanatomy of early visual areas, and suggest that investigating pRFs in TS may shed insights into their atypical visuospatial processing.

Key words: retinotopy; sex differences; Turner syndrome; visual cortex; visuospatial cognition

\section{Significance Statement}

Turner syndrome is caused by the absence of one of the two X-chromosomes in females. Using functional neuroimaging and population receptive field mapping, we find that chromosome dosage variation (X-monosomy) associated with Turner syndrome affects the functional neuroanatomy of the visual cortex. Specifically, girls with Turner syndrome have smaller early visual areas that provide lesser coverage of the peripheral visual field compared with healthy controls. Our observations provide compelling evidence that the $\mathrm{X}$-chromosome affects not only parietal cortex, as described in previous studies, but also affects early visual areas. These findings suggest a paradigm change in understanding the effect of $\mathrm{X}$-monosomy on the development of visuospatial abilities in humans.

\section{Introduction}

Turner syndrome (TS) is a genetic condition in females (prevalence of 1:2000) caused by the absence of most or all of one
X-chromosome (Stochholm et al., 2006). TS is characterized by a specific cognitive profile with relative strengths in verbal domains and weaknesses in visuospatial and executive processing (Hong et al., 2009). Compared with typically developing controls, structural imaging of the brain shows smaller gray matter volume and

the data. We thank all the girls and families who kindly volunteered to participate; and the Turner Syndrome Society and the Turner Syndrome Foundation, which made this work possible.

The authors declare no competing financial interests.

*K.G.-S. and A.L.R. contributed equally to this work.

Correspondence should be addressed to Tamar Green at tgreen2@stanford.edu.

https://doi.org/10.1523/JNEUROSCI.2745-18.2019

Copyright $\odot 2019$ the authors 
surface area and larger thickness of the parieto-occipital cortex in prepubertal and adolescent girls as well as adults with TS (Raznahan et al., 2010; Lepage et al., 2013; Green et al., 2014). Beyond these anatomical differences, resting-state and task-based fMRI studies find aberrant brain activity in the dorsal visual stream (Mishkin et al., 1983) of girls with TS (Kesler and Reiss, 2006; Green et al., 2018). Despite the abundance of evidence indicating dorsal visual stream abnormalities in TS, it is unknown whether aberrant processing occurs in early visual areas.

We hypothesize that behavioral differences in visuospatial processing between TS and controls may be a result of differences in spatial processing by neurons in the visual system. We will consider three scales of spatial processing: the scale of a neuron, the scale of a population of neurons within a voxel, and the scale of an entire visual area.

In the visual system, each neuron can be characterized by its receptive field, that is, the region in the visual field in which the neuron processes visual information (Hubel and Wiesel, 1998). Thus, the receptive field is the fundamental spatial processor in the visual system. Neurons whose receptive fields cover adjacent locations in the visual field are also physical neighbors in the cortex, and therefore are clustered. The scale of this clustering in the human brain is a few millimeters. Thus, we can measure with fMRI the population receptive field ( $\mathrm{pRF}$ ), namely, the part of the visual field processed by the population of neurons within a voxel (Dumoulin and Wandell, 2008). Additionally, neurons in visual cortex are organized in visual field maps referred to as visual areas (Wandell and Winawer, 2011). In each hemisphere, pRFs in each of the early visual areas tile the contralateral half of the visual field.

Recent developmental studies report that $\mathrm{pRF}$ and visual field coverage (VFC) in early visual areas V1-V3 are adult-like in typically developing children as young as $5-7$ years (Gomez et al., 2018; Dekker et al., 2019), even as some aspects of visuospatial processing continue to develop (e.g., Vernier acuity, a measure of cortical positional resolution, is adult-like only in 10- to 14-yearolds) (Carkeet et al., 1997; Skoczenski and Norcia, 2016). These findings allow us to investigate whether spatial processing by pRFs in early visual cortex is different in TS compared with controls already during childhood. Specifically, we hypothesized that the relative weakness in visuospatial processing observed in TS may be due to poorer spatial pooling and integration stemming from one or more factors: (1) atypical arrangement and/or size of visual areas, (2) smaller pRF size, (3) more centralized (lower) pRF eccentricity, (4) atypical pRF size versus eccentricity relations, and (5) atypical coverage of the visual field.

To test these hypotheses, we measured and compared pRFs and VFC in 20 girls with TS and 22 typically developing age- and sex-matched controls. We considered two possible main outcomes: (1) finding between-group differences in pRF properties/ VFC would provide evidence for atypical spatial processing in TS originating in early visual cortex; or (2) finding no betweengroup differences would suggest that visuospatial deficits characteristic of TS are likely due to differences in spatial processing in higher-level regions of the parietal cortex.

To test the relation between processing in early visual cortex and behavior, we also examined whether the properties of early visual areas (size of visual areas, pRF size, and pRF eccentricity) correlate with a visuospatial task (Picture Puzzles task from the NEuroPSYchological test, version II, [NEPSY-II]). Finding a correlation between properties of early visual areas and performance in the visuospatial task would support the prediction that aberrations in early visual areas have behavioral consequences. Thus, the goal of this research is to enhance understanding of visual cortical development and function in a unique, female-only patient population.

\section{Materials and Methods}

\section{Study design}

To test our hypotheses, we used fMRI to measure and evaluate $\mathrm{pRFs}$ in each voxel in girls with TS and, age- and sex-matched controls. Participants were scanned as they viewed a flickering checkerboard bar that swept the visual field while fixating on a central stimulus and indicating when its color changed via a button press. We first examined whether there are qualitative differences between girls with TS and controls in polar angle and eccentricity maps in the visual areas. Then, we examined whether there are quantitative differences in pRF properties (size, eccentricity, and size vs eccentricity relations) between girls with TS and controls. Finally, we tested whether there are qualitative or quantitative differences between TS and controls in the VFC of early visual areas.

\section{Participants}

A total of 52 human females completed pRF mapping: 24 girls with TS, ages 7.1-13.8 years (10.4 \pm 2.0$)$ and 28 typically developing controls, ages $7.0-13.2$ years $(10.2 \pm 1.9)$. We assessed visual acuity before participation in the study. If myopia/hyperopia was reported, we provided MRI safe glasses to provide satisfactory visual acuity.

Participants with TS were recruited through the National Turner Syndrome Society and the Turner Syndrome Foundation, a local network of physicians, and advertisement on the Stanford University School of Medicine website. Control participants were recruited through local print media and parent networks. Exclusion criteria for both groups included premature birth (gestational age $<34$ weeks), low birth weight $(<2000 \mathrm{~g})$, and known diagnosis of a major psychiatric condition (i.e., psychotic or mood disorder) or neurological disorder, including seizures. All participants in the TS group had X-monosomy, confirmed by karyotype reports supplied by families or through medical records. Girls with TS exhibiting mosaic or uncommon structural karyotypes were excluded. The Institutional Review Board of the Stanford University School of Medicine approved this study, and informed written consent was obtained from a legal guardian for all participants. Written assent was obtained from participants 7 years of age and older.

\section{MR data acquisition}

A total of 52 participants underwent pRF mapping in a 3T MRI scanner. Participants were scanned as they viewed either expanding rings or rotating wedges filled with black-and-white flickering checkerboards. Participants were instructed to fixate at all times on a central stimulus and indicate when its color changed via a button press.

To decrease the likelihood of poor scan quality due to excessive motion in the scanner, a common issue in pediatric neuroimaging (Grill-Spector et al., 2008), participants underwent behavioral training in a mock MRI scanner before their actual scan. This was done to desensitize them to the appearance and sounds of an MRI environment and to help them learn to limit motion.

All MR data were collected at the Lucas Center for Imaging at Stanford University using a 3 tesla GE Discovery MR 750 scanner. For structural data, we used a fast-spoiled gradient recalled echo pulse sequence to obtain a high-resolution $\mathrm{T} 1$ anatomical brain image for each participant (176 sagittal slices, $\mathrm{TR}=8.2 \mathrm{~ms}, \mathrm{TE}=3.2 \mathrm{~ms}$, inversion time $=450 \mathrm{~ms}$, flip angle $=12^{\circ}$, number of excitations $=1, \mathrm{FOV}=240 \times 192 \mathrm{~mm}$; matrix $=256 \times 256$; voxel size $=1.0 \times 1.0 \times 1.0 \mathrm{~mm}$ thickness, acquisition time $=4 \mathrm{~min} 29 \mathrm{~s}$ ).

We collected BOLD data using an 8-channel phased-array surface coil (Nova Medical). A total of 32 slices oriented horizontally to anterior commissure-posterior commissure line were collected using a $\mathrm{T}^{*}$ weighted gradient echo spiral pulse sequence with $\mathrm{TR}=2,500 \mathrm{~ms}, \mathrm{TE}=$ $30 \mathrm{~ms}$, flip angle $=77^{\circ}, \mathrm{FOV}=192 \mathrm{~mm}$. Functional data were collected with $3.00 \mathrm{~mm}$ isotropic voxels with no gaps between slices. Visual stimuli were projected onto a screen and viewed through an angled mirror mounted above the supine participant's head. 


\section{Stimuli}

Retinotopy stimuli were standard ring and wedge stimuli described previously (Witthoft et al., 2014). Subjects participated in two runs in which we measured polar angle using a $45^{\circ}$ wedge, rotating clockwise about fixation at a rate of $22.5^{\circ}$ every $2.5 \mathrm{~s}$, and two runs in which we measured eccentricity, using a series of expanding concentric rings (1.4 $4^{\circ}$ in width) centered at fixation. The innermost ring consisted of a disk with a diameter of $\sim 2.8^{\circ}$, whereas the largest ring extended $14^{\circ}$ from fixation. Both the wedge and ring stimuli contained $100 \%$ contrast black-and-white checkerboards, which reversed contrast at a rate of $4 \mathrm{~Hz}$. Each run contained six cycles (either a full rotation of the wedge or a full expansion of the ring), each lasting $40 \mathrm{~s}$, which were interspersed with four $20 \mathrm{~s}$ blank periods. In addition, each scan began and ended with a blank $10 \mathrm{~s}$ block. Thus, we acquired 120 TRs per run. Instructions: To maintain attention to the stimuli, participants were instructed to fixate on a central dot and press a button when its color changed.

We modeled the pRF of each voxel as a 2D Gaussian followed by a nonlinearity, referred to as compressive spatial summation (Kay et al., 2013, 2015). Compressive spatial summation improves pRF fits in higher-level visual areas (Kay et al., 2013, 2015). pRFs were fit to the time series data of each voxel using the implementation in vistasoft: https:// github.com/vistalab/vistasoft (Dumoulin and Wandell, 2008). For each voxel, the model estimates the pRF center and its size in degrees of visual angle as well as its gain factor and compressive summation (exponent typically $<1$ ).

\section{Behavioral data acquisition}

Subtests from the NEPSY-II (Korkman et al., 1998) were used for assessment of visuospatial processing. Specifically, we administered the Picture Puzzles subtest from the NEPSY-II battery. The Picture Puzzles subtest is designed to test visual discrimination, spatial localization, visual scanning, and the ability to construct and reconstruct part-whole relationships. To test for associations between responses in early visual cortex and behavior, we assessed correlations between performance on the Picture Puzzles subtest, which provides a broad assessment of visuospatial abilities, with functional properties of early visual cortex. In short, this task is similar to a picture puzzles game. We ask the child to scan a large picture with a superimposed grid dividing the picture into rectangles. Next, to the large picture, the child sees some pieces from the same picture ("puzzle piece"). We then ask the child to point to the area in the picture where the puzzle piece belongs. The Picture Puzzles test assesses the nonmotor aspect of visual perception. Performance of this task requires visual scanning, visual perception, and visual comparison of details. This task does not test for the visuoconstruction (the coordination of fine motor skills with spatial abilities) or visuomotor functions required for tasks, such as design copying or block construction.

We used the standard scores according to the participant's age for the Picture Puzzles test provided in the NEPSY-II manual. Standard scores have a mean of 10 (SD 3, range 1-19).

To provide a general assessment of cognitive abilities, we also administered the Wechsler Intelligence Scale for Children Edition 4 (Wechsler, 2003). We recorded the Full-Scale Intelligence Quotient scores (scaled for age and sex), and composite scaled scores of the Verbal Comprehension Index, Perceptual Reasoning Index, Working Memory Index, and Processing Speed Intelligence scores.

\section{Data analysis}

Anatomical data analysis. All images were manually inspected and rated separately for image quality by two raters. In cases where the raters did not agree on acceptable image quality, the final decision for exclusion was reviewed with an expert rater (K.G.-S.). Cortical reconstruction was performed using FreeSurfer version 5.3 (http://surfer.nmr.mgh.harvard. $\mathrm{edu} /$ ). All scans were preprocessed using bias field correction methods available with SPM8 (http://www.fil.ion.ucl.ac.uk/spm) before using the FreeSurfer pipeline. Image editors visually inspected the gray-white and pial surfaces, and when needed, performed appropriate manual corrections as per the FreeSurfer Tutorial (http://surfer.nmr.mgh.harvard.edu/ fswiki/FsTutorial). Gray-white segmentation corrections were made manually after the FreeSurfer automatic segmentation using editing tools in ITK-snap (http://www.itksnap.org/pmwiki/pmwiki.php). All image editors were trained to achieve interrater reliability of $\geq 0.95$ (intraclass correlation coefficient) for ROIs using gold-standard datasets developed in our laboratory. The manually corrected segmentation was then processed by FreeSurfer to generate a cortical surface reconstruction at the gray-white boundary of each participant's brain. Cortical visualizations on inflated meshes were then done in mrVista (https://github.com/vistalab/vistasoft).

We used the anatomical data to compute the surface area and cortical thickness (CT) of V1-V3 (Dumoulin and Wandell, 2008). The data were extracted using FreeSurfer and computed separately for the dorsal and ventral parts of V1, V2, and V3 for consistency with the retinotopy analyses. For the surface area, we summed the dorsal and the ventral portion of each area (e.g., V2 dorsal + V2 ventral). The CT computed by Freesurfer is an average of the CT at each vertex in the visual area (e.g., V2d), so a sum of dorsal and ventral areas is not applicable. Thus, for each visual area (V1-V3), we weighted CT by surface area (i.e., calculated the combined CT average across the ventral and the dorsal parts of each visual area). For example, for left V2, we calculated L_V2 thickness $=\left(\left(L_{-}\right.\right.$V2d thickness $\times$ L_V2d surf area $)+($ L_V2v thickness $\times$ L_V2v surf area $))$ / (L_V2d surf area + L_V2v surf area) (https://surfer.nmr.mgh.harvard. edu/fswiki/UserContributions/FAQ).

\section{fMRI data analysis}

Estimating $p R F s$. After functional data were transformed to the wholebrain anatomy and restricted to the cortical ribbon, a pRF model was fit for each voxel (Dumoulin and Wandell, 2008). For each voxel, a 2D Gaussian receptive field was modeled. The pRF model estimates 5 variables at the voxel level: a center described by $x(1)$ and $y(2)$ coordinates, (3) a size, which is the SD of the Gaussian $(\sigma),(4)$ a gain parameter $(\mathrm{g})$, and (5) a compressive nonlinearity (exponent, $n<1$ ). The model is solved in two steps: First, a grid search is used by testing candidate pRF predictions versus the data. The candidate time course is produced by taking pRF parameters, and then convolving a hemodynamic response function with the product of the stimulus movie and the pRF. We generate many $(\sim 100,000)$ candidate pRFs using a range of $x, y$, and $\sigma$ values, and find the parameters that best explain the variance of the voxel's time course. Second, after finding the best parameters from the grid search, we optimize each of the parameters using optimization tools in MATLAB (The MathWorks) starting from the grid search estimates to get the final pRF parameters for each voxel. Voxels were included for subsequent analyses if the variance explained by the pRF model was $>5 \%$. This is a liberal threshold designed to exclude voxels that are not modulated by the stimulus.

\section{Visual field maps}

V1-V3 were defined and drawn following widely acceptable criteria reviewed by Wandell et al. (2007). When we draw the boundaries of these areas, we follow the phase map (the vertical meridian is the boundary between V1 and V2, and the horizontal is the boundary between V2 and V3). An independent rater (K.G.-S.), who was blind to the group diagnosis of each participant, examined the definition of functional regions and corrected them, in case the V1-V3 definition did not follow the above criteria. The dorsal and ventral parts of V2 and of V3 were combined into a single map. Visual field delineations in all subjects are shown in Figure 1.

\section{$p R F$ size versus eccentricity fits}

To evaluate the relationship between a pRF's size and its eccentricity, voxels within each visual area of each participant were entered into a linear regression relating $\mathrm{pRF}$ size versus eccentricity. In the linear regression, each voxel's contribution was weighted by the variance explained of the pRF model. The line of best fit was derived in each subject for each area. Additionally, to ensure the most accurate pRF fits, voxels whose $\mathrm{pRF}$ centers were $>10^{\circ}$ radial eccentricity were not included, as the data $>10^{\circ}$ radial eccentricity was too sparse to allow accurate estimation.

\section{VFC analyses}

To calculate the VFC for a given visual field map and subject, all voxels in an area that contain pRFs with $>5 \%$ variance explained by the model are included and modeled as a Gaussian with a peak normalized to 1 . The 
Table 1. Demographics and clinical characteristics ${ }^{a}$

\begin{tabular}{lccc}
\hline & TS & Controls & $p$ \\
\hline No. of participants & 20 & 22 & - \\
Age, yr & $10.3(2.0)$ & $10.4(1.9)$ & NS \\
Age range, yr & $7.0-13.1$ & $7.0-13.8$ & - \\
Sex & Female & Female & - \\
Tanner stage ${ }^{b}$ & pubic (breast) & pubic (breast) & NS \\
$\quad \leq 2$, & $17(20)$ & $16(17)$ & - \\
3 & $3(1)$ & $3(2)$ & - \\
$\quad 4$ & $0(0)$ & $3(3)$ & - \\
$\quad 5$ & $0(0)$ & $0(0)$ & $<0.001$ \\
FSIQ & $96.0(12.3)$ & $116.9(10.9)$ & $<0.01$ \\
VCI & $105.5(13.1)$ & $117(13.6)$ & $<0.001$ \\
PRI & $97.3(13.8)$ & $115.4(11.3)$ & $<0.01$ \\
PSI & $88.8(15.9)$ & $105.9(15.4)$ & $<0.001$ \\
WMI & $91.4(12.0)$ & $109.4(13.7)$ & $<0.01$ \\
Picture Puzzles scaled & & $10.6(3.7)$ & \\
\hline
\end{tabular}

${ }^{a}$ Values are mean $\pm S D$. Welch two-sample $t$ test was used to assess significance between groups. FSIQ, Full-Scale Intelligence Quotient; VCI, Verbal Comprehension Index; PRI, Perceptual Reasoning Index; PSI, Processing Speed Intelligence; WMI, Working Memory Intelligence.

${ }^{b} p=0.38$ for pubic hair and $p=0.08$ for breast development on Tanner stages of development for females (Fisher's Exact Test, given the small number of participants with Tanner Stages 3 and 4).

$c n=18$ for the TS group.

VFC is produced at each point by averaging the value across pRFs that cover that point, and then normalizing by the maximum coverage value in that subject. We also implemented a bootstrapping procedure that draws with replacement $n$ voxels from a subject's maps of size and produces an average VFC from 50 iterations to reduce the effect of outlier voxels. The average VFC from this bootstrapping approach is the VFC used for a given subject's ROI.

\section{Statistical analysis}

Statistical analysis was conducted using R (https://www.r-project.org/). We used a $t$ test to test for group differences in demographic characteristics. Three-way ANOVAs with factors of group, hemisphere, and visual areas (V1-V3) were run for, surface area, CT, visual areas size, pRF size, and pRF eccentricity as separate variables. For all statistical tests, we report significant main effects or interactions. We used $t$ test for post hoc between-group comparisons. Specifically, the Welch two-sample $t$ test with degrees of freedom adjusted was applied since this test does not assume equal variance.

To estimate the relationship between a pRF's size and eccentricity, we estimated the linear regression between $\mathrm{pRF}$ size and eccentricity for each voxel across visual field maps for each subject. The line-of-best fit was derived in each participant, and then the slope and intercept of this line were averaged across the TS group and the control group. We used a $t$ test to compare the slope and intercepts between the groups. In Figure 2D, we show the average slope and intercept of this line across participants of each group.

For our analysis of between-group differences in pRF properties, we conducted brain-behavior correlations (Pearson correlation). We also tested whether these correlations differed between the groups using the Fisher's test. Given the exploratory nature of the brain-behavior correlation analyses, we did not correct for multiple comparisons.

\section{Results}

\section{No quantitative differences between data quality in TS versus controls}

Of the 52 participants, 4 girls with TS and 6 controls were excluded based on their poor task performance during fMRI. Accordingly, data from 20 girls with TS, ages 7.0-13.1 years (mean age, $10.3 \pm 2.0)$ and 22 age- and sex-matched controls ages 7.013.8 years (mean age $10.4 \pm 1.9$ ) were analyzed (Table 1 ).

We conducted quality control analyses for all remaining 42 participants to ensure that any observed between-group effects were not due to motion, performance during fMRI, goodness of pRF model fits.

Quality control analyses indicated that: (1) there were no significant between-group differences in motion (TS motion average: $1.5 \pm 1.7 \mathrm{~mm}$; controls: $1.7 \pm 1.1 \mathrm{~mm} ; t_{(31)}=0.44, p=$ $0.663)$; (2) there were no between-group differences in overall task performance during fMRI $\left(t_{(28)}=-1.9, p=0.068\right)$, as well as no between-group differences in each of the fMRI tasks (expanding rings, two runs $t_{(21.5)}=-1.9, p=0.071 ; t_{(23.4)}=-1.5$, $p=0.147$; and rotating wedges, two runs $t_{(36.1)}=-1.6, p=$ $\left.0.118 . t_{(35.6)}=-1.5, p=0.142\right)$; and (3) there were no significant between-group differences in the percentage of variance explained by the pRF model across early visual areas $\left(F_{(1,147)}=\right.$ $0.001, p=0.975)$.

\section{Qualitatively comparable visual areas in TS and controls}

Prior studies in both typical adults and children show that, in each of the early visual areas: (1) pRFs generate a topographic map of the visual field via approximately orthogonal representations of polar angle (angle from horizontal axis) and eccentricity (distance from fixation) (Sereno et al., 1995); (2) pRF size increases approximately linearly with eccentricity (Wandell and Winawer, 2011); (3) mean pRF size increases across the hierarchy of visual areas; the smallest pRFs are present in V1 and progressively increase in size in subsequent visual areas (Wandell and Winawer, 2015; Gomez et al., 2018; Dekker et al., 2019); and (4) pRFs in each area tile the visual field, providing in each hemisphere a hemifield representation of the contralateral visual field (Holmes, 1945; Connor et al., 2004; Gomez et al., 2018; Dekker et al., 2019). Here, we compared these properties across girls with TS and age- and sex-matched typically developing controls.

We first examined whether there are qualitative differences between girls with TS and controls in polar angle and eccentricity maps as well as whether there are differences in the sizes of visual areas. Then, we examined whether there are quantitative differences between girls with TS and controls in pRF properties (pRF size, $\mathrm{pRF}$ eccentricity, and $\mathrm{pRF}$ size vs eccentricity relations). Finally, we tested whether there are qualitative or quantitative differences between TS and controls in VFC.

Examination of the topographic organization of polar angle and eccentricity maps showed that it was qualitatively similar across groups (Fig. 1). That is, girls with TS, like controls, displayed a series of mirror-reversed polar angle maps (Fig. 1A,C) emerging in and around the calcarine sulcus (where V1 is located). Eccentricity progressively increased along a posterior to anterior axis (Fig. $1 B, D$ ), spanning the medial aspect of the occipital cortex. Using these polar angle and eccentricity maps, we defined visual field maps V1 through V3 bilaterally in each individual using standard methods (Sereno et al., 1995; Wandell et al., 2015). These regions were defined in each of the participants' brains (20 with TS; 22 controls; Fig. 1) and spanned the central $10^{\circ}$ of visual angle.

\section{Smaller V1-V3 in TS versus controls}

After defining V1-V3 in each participant's brain, we measured the cortical volume of these visual field maps. We used a threeway ANOVA with factors of group, hemisphere, and area (V1V3) to test for differences in cortical volume. While there were no significant between-group differences in total brain volume $\left(t_{(39.2)}=-0.86, p=0.395\right)$, results showed that the cortical volume of early visual field maps V1-V3 was significantly smaller in girls with TS compared with controls $\left(F_{(1,247)}=16.07, p=\right.$ 8.08e-05; Fig. 2A). We then used the Welch two-sample $t$ test to 


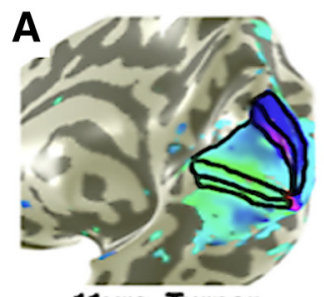

11yrs, Turner

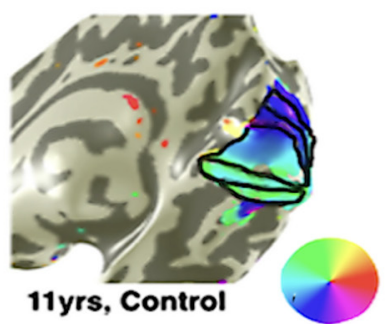

Turner, phase

C
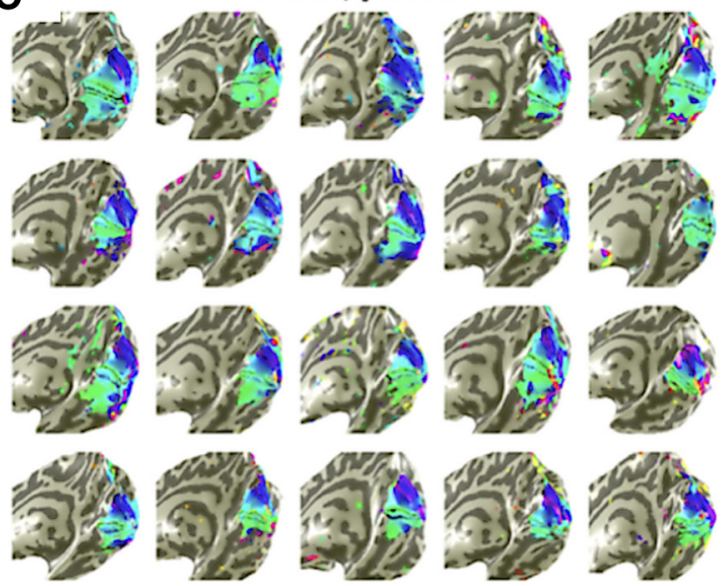

Control, phase
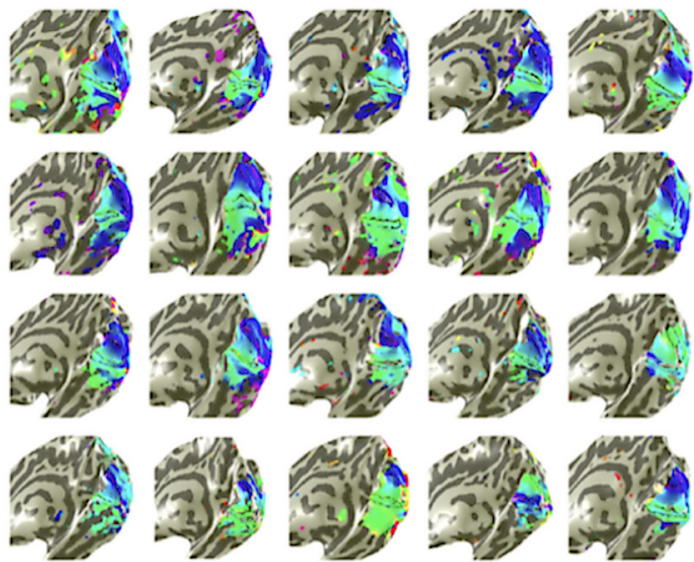
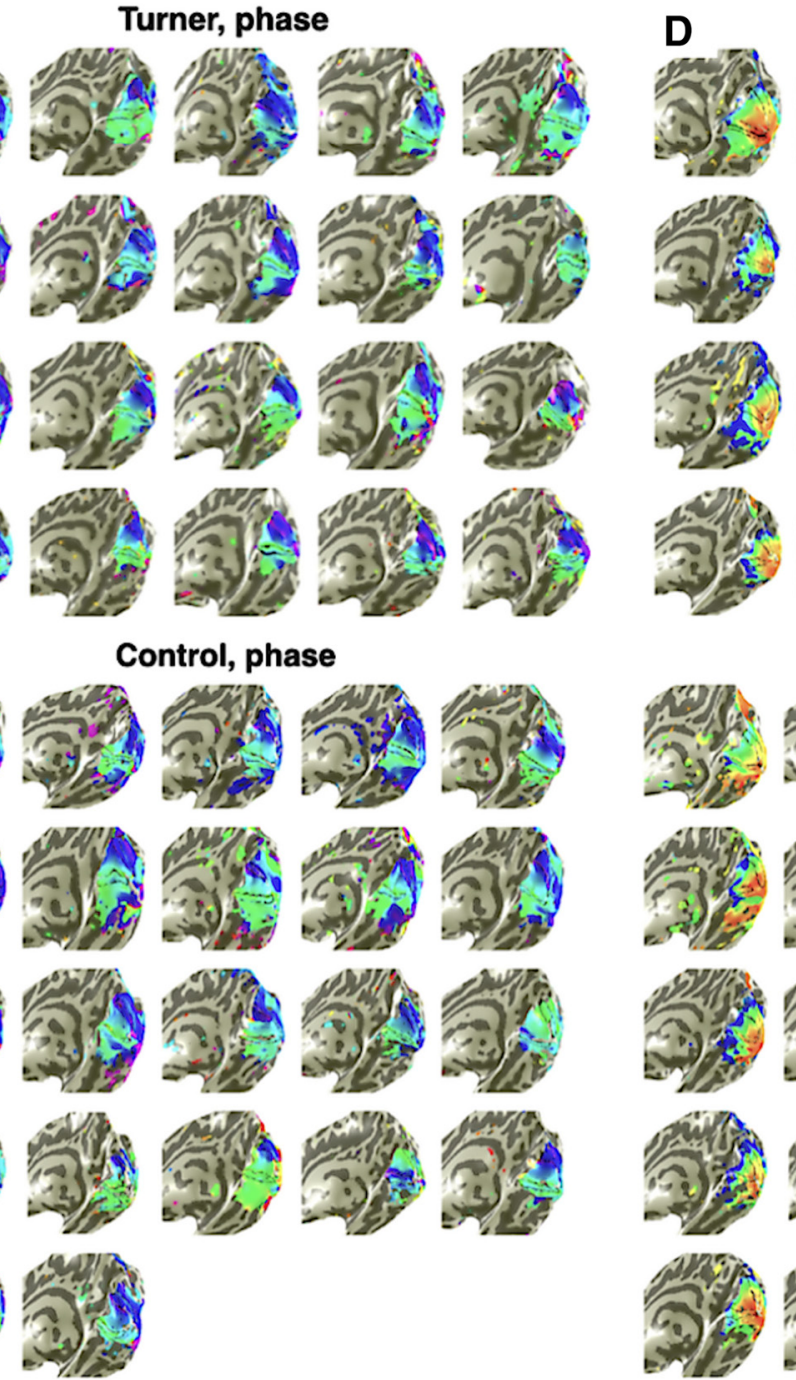

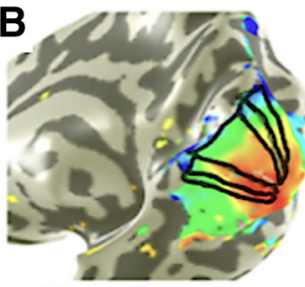

11yrs, Turner

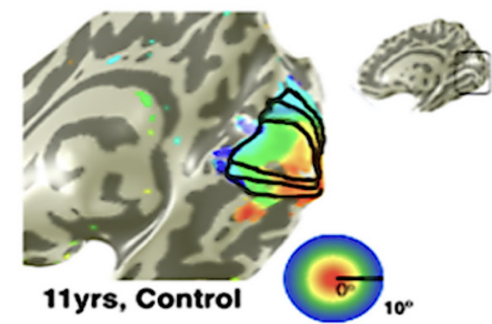

Turner, eccentricity
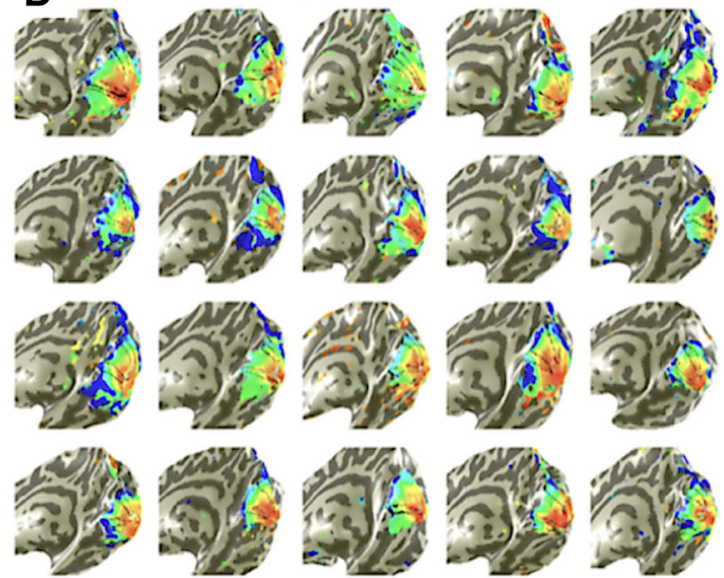

Control, eccentricity
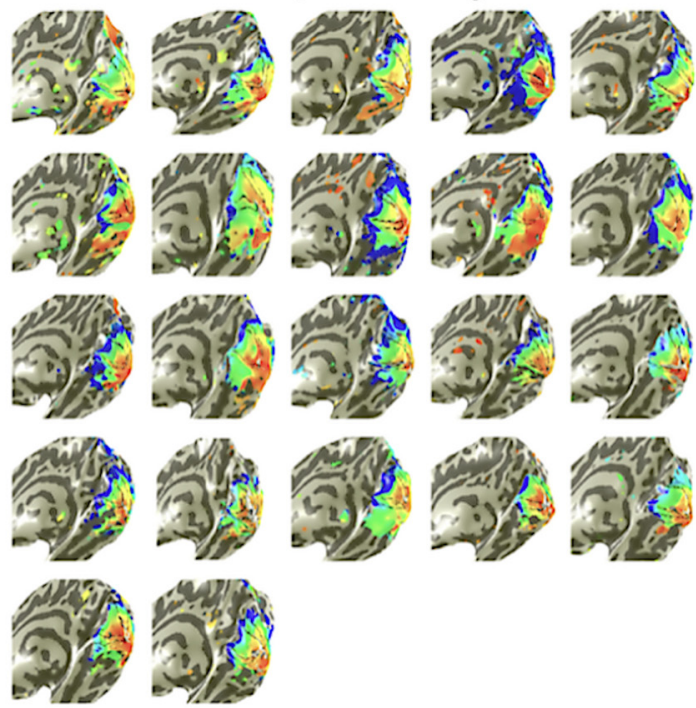

Figure 1. Polar angle and eccentricity maps are qualitatively similar in girls with TS and sex-and age-matched controls. $\boldsymbol{A}$, Polar angle maps on the right visual cortex from an example 11 -year-old girl with TS and an example 11-year-old typically developing girl. Color wheel represents polar angle. $\boldsymbol{B}$, Eccentricity maps in the same subjects. Color wheel represents eccentricity. Lines along the visual cortex were drawn marking the boundaries of visual maps: V1, V2 dorsal and ventral, and V3 dorsal and ventral. Polar angle maps ( $\boldsymbol{C}$ ) and eccentricity maps ( $\boldsymbol{D}$ ) in the right hemisphere of all participants. Top, TS. Bottom, Controls. Maps represent the central $10^{\circ}$ and are thresholded at $5 \%$ variance explained, voxel-level.

determine whether the mean V1-V3 cortical volume was different between TS and control groups. Between-group differences in cortical volume were significant in left V1 $\left(t_{(32.3)}=2.3, p=\right.$ $0.026)$, right $\mathrm{V} 2\left(t_{(39.5)}=3.0, p=0.005\right)$, and right V3 $\left(t_{(38.6)}=\right.$ $2.8, p=0.007$; Fig. $2 A)$. As expected, in both groups, the size of visual areas progressively decreased from V1 to V2 to V3 $\left(F_{(2,247)}=\right.$ 121.9, $p=1.48 \mathrm{e}-37$; Fig. $2 A)$. Furthermore, results showed that the cortical volume of early visual field maps was smaller in the left hemisphere compared with the right, independent of group and area $\left(F_{(1,147)}=6.8, p=0.010\right.$; Fig. $\left.2 A\right)$. Thus, the cortical volume of early visual areas defined by retinotopic mapping of the visual field is reduced in TS.
To determine whether our finding of lower cortical volume in TS is due to lower surface area, lower CT, or both, we compared the surface area and CT estimated using FreeSurfer tools from the structural imaging data across groups. We used these structural data, as complementary, independent information on early visual areas development. Results showed that the cortical surface area of early visual field maps V1-V3 was significantly smaller in girls with TS compared with controls $\left(F_{(1,247)}=32.2, p=3.88 \mathrm{e}-08\right.$, three-way ANOVA with factors of group, hemisphere, and area). We then used $t$ tests to determine in which of the early visual areas was the surface area different between TS and controls. We found significant between-group differences in surface areas in bilateral 

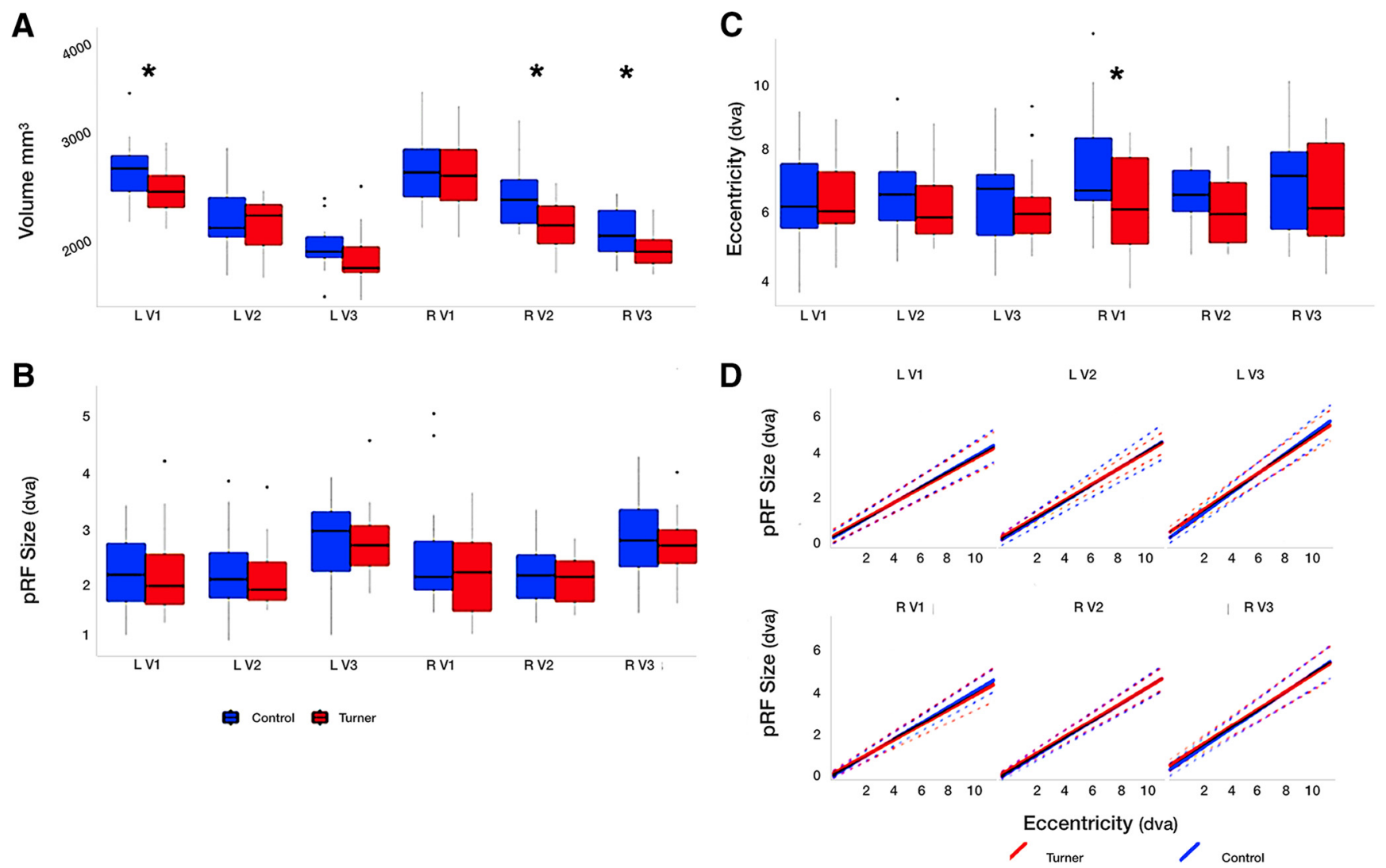

Figure 2. Visual maps differ in size and pRF differ in eccentricity between TS and controls. $\boldsymbol{A}$, Mean cortical volume of V1, V2, and V3 in TS (red) ( $n=20)$ and controls (blue) ( $n=22)$. $\boldsymbol{B}$, Mean population receptive field (pRF) size in TS versus controls. $C$, Mean pRF eccentricity in TS versus controls. $A-C$, Boxplots represent the following information. Black horizontal line indicates median. Box represents interquartile range. Vertical lines indicate maximum and minimum scores, which are no more than 1.5 times the interquartile range. ${ }^{*} p<0.05$, significant differences between groups. D, pRF size versus eccentricity relationship in TS and controls. The line of best fit (solid line) and the SE (dashed line) indicate the relationship between pRF eccentricity and size in units of degrees of visual angle (dva). Fits are calculated in each participant; slopes and intercepts are then averaged across participants.

V1 and V2 (left V1, TS, $1455 \pm 227 \mathrm{~mm}^{2}$, controls, $1698 \pm 266$ $\mathrm{mm}^{2}, t_{(39.9)}=-3.20, p=0.003$; right V1, TS, $1434 \pm 214 \mathrm{~mm}^{2}$, controls, $1601 \pm 292 \mathrm{~mm}^{2}, t_{(36.42)}=-2.22, p=0.033$; left V2, TS, $1359 \pm 190 \mathrm{~mm}^{2}$, controls, $1524 \pm 156 \mathrm{~mm}^{2}, t_{(34.6)}=-2.83$, $p=0.008$; and right V2, TS, $1148 \pm 167 \mathrm{~mm}^{2}$, controls, $1542 \pm$ $\left.146 \mathrm{~mm}^{2}, t_{(38.0)}=-2.35, p=0.024\right)$. In contrast, there was no significant between-group difference in CT of early visual field maps V1-V3. Together, results indicate that the smaller volume of V1-V3 in TS versus controls stems from smaller cortical surface area.

\section{Smaller pRF eccentricity in TS versus controls}

We next measured mean pRF size and pRF eccentricity in V1-V3. We used three-way ANOVA with factors of group, hemisphere, and area to test for differences in pRF properties. Results showed that mean pRF size of V1-V3 did not differ between TS and controls $\left(F_{(1,147)}=0.6, p=0.437\right.$; Fig. $\left.2 B\right)$. In both groups, $\mathrm{pRF}$ size progressively increased from V1/V2 to V3 $\left(F_{(2,147)}=19.2\right.$, $p=1.72 \mathrm{e}-08$; Fig. $2 B$ ). In contrast, mean pRF eccentricity of early visual areas V1-V3 was significantly lower in girls with TS compared with controls $\left(F_{(1,247)}=8.1, p=0.005\right.$; Fig. $\left.2 C\right)$. The Welch two-sample $t$ test was used to determine whether mean pRF eccentricity in TS and controls was different from each other. Between-group differences were significant in right V1 $\left(_{(39.9)}=\right.$ $2.1, p=0.042)$, and trended in the same direction in V2, bilaterally (left $t_{(39.5)}=1.6, p=0.118$; right $t_{(37.8)}=1.7, p=0.097$; Fig. $2 C)$. Finally, in both groups and in each visual area, $\mathrm{pRF}$ size was linearly related to eccentricity, with $\mathrm{V} 3$ showing a steeper slope than V1/V2, and no significant between-group differences (Fig. $2 D)$. Thus, retinotopic organization and $\mathrm{pRF}$ size versus eccentricity relationships in $\mathrm{V} 1-\mathrm{V} 3$ are similar across TS and controls; however, mean pRF eccentricity is lower (closer to the center of gaze) in TS than controls.

\section{Qualitatively comparable VFC in TS and controls}

VFC was obtained by evaluating how the collection of pRFs spanning each visual field map (across both hemispheres) tiles the visual field. VFC of V1-V3 showed no qualitative between-group differences (Fig. 3). In V1-V3, the VFC spanned a hemifield in each hemisphere in both TS and controls. As there were also no qualitative differences in polar angle and eccentricity maps between TS and controls (Fig. 1), together, these analyses reveal that girls with TS have similar polar angle and eccentricity maps as well as VFC in V1-V3 relative to controls.

\section{Scarcity of pRF centers in the peripheral visual field in TS versus controls}

To further examine what drives the observed differences in mean pRF eccentricity between TS and controls in early visual areas, we plotted the location of pRF centers in the visual field (Fig. 4A) and then tested whether there were between-group differences in the distribution of pRF centers across eccentricity bands $\left(0^{\circ}-4.99^{\circ}\right.$, $5^{\circ}-9.99^{\circ}$, and $10^{\circ}-15^{\circ}$ ).

Results show that pRF centers span the visual field across all three eccentricity bands in both TS and controls. However, there were less pRF centers in the peripheral visual field $\left(10^{\circ}-15^{\circ}\right)$ in TS 

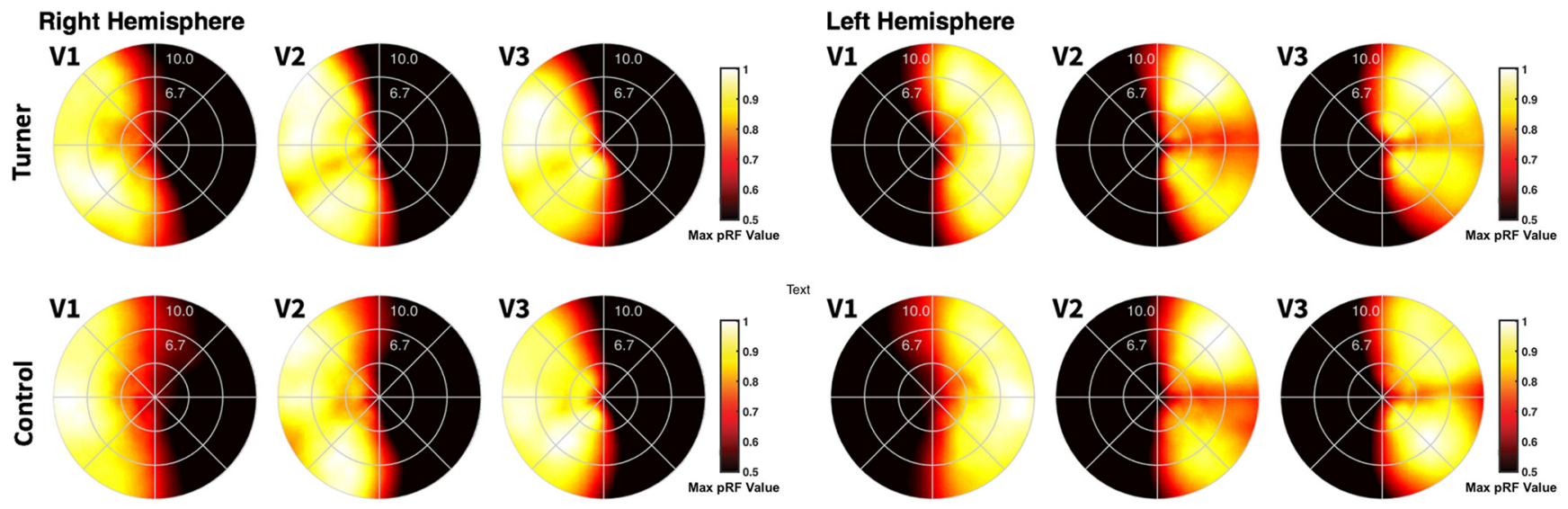

Figure 3. Visual field coverage is qualitatively similar in girls with Turner Syndrome and controls. Visual field coverage of V1-V3 computed using the average maximum pRF density coverage for each subject and then averaged across subjects from each group. Maps are shown for the left and right hemisphere data. Top, Turner Syndrome. Bottom, Controls. Inner to outermost ring segments correspond to 3.4, 6.7, and 10 degrees of visual angle (dva), respectively. Color bar represents average maximum pRF coverage.

than controls (Fig. 4A). Quantification of the number of pRFs per eccentricity band reveals no significant differences between groups in the number of pRFs in either the $0^{\circ}-4.99^{\circ}\left(t_{(40)}=0.96\right.$, $p=0.343)$ or $5^{\circ}-9.99^{\circ}\left(t_{(33.1)}=0.38, p=0.706\right)$ bands. However, across $\mathrm{V} 1-\mathrm{V} 3$, there were significantly less pRFs covering the peripheral band $\left(10^{\circ}-15^{\circ}\right)$ in girls with TS than controls $\left(t_{(39.8)}=\right.$ 2.2, $p=0.034$, not corrected; TS, mean $=2338 \pm 798$; controls, mean $=2871 \pm 798$; Fig. $4 B$ ).

We next tested whether the lower number of pRF centers in the periphery in TS than controls is due to less pRFs in V1-V3 of TS than controls or due to a difference in the distribution of $\mathrm{pRF}$ into the periphery. To test these possibilities: (1) we examined whether there are significant differences in the overall number of pRFs across groups; and (2) we used kernel density estimation (KDE) on pRF locations to test whether the density of pRF in the peripheral visual field is lower in TS than controls. We found a nonsignificant trend for overall fewer pRFs in TS than controls across these three eccentricity bands of early visual areas (TS, mean $=13,809 \pm 2134$, controls mean $=14,993 \pm 2313, t_{(39.9)}=$ 1.7, $p=0.097$, not corrected). KDE revealed that, while both groups show the same pattern of denser coverage of the visual field by pRFs in the center compared with the periphery, the density pattern of the $\mathrm{pRF}$ centers is more centralized around the fovea in TS than controls (Fig. 4C). These findings indicate that fewer pRFs are allocated to the periphery of the visual field in TS relative to controls.

\section{Mean pRF sizes and eccentricity correlate with visuospatial performance in TS, but not in typical controls}

To examine whether pRF properties may impact visual processing, we conducted an evaluation of visual abilities of scanning, processing, and comparison of details using the Picture Puzzles task from the NEPSY-2 (Korkman et al., 1998). In this task, a large picture was shown with a grid superimposed, dividing the picture into rectangles. Parts of the large picture, "puzzle picture," were presented on the side of the large picture. Participants were asked to identify where each of these rectangles belonged in the larger picture. Scores range from 1 (well below expected level) to 19 (superior). On average, girls with TS performed at a borderline level $(6.9 \pm 3.5)$, and significantly worse than controls $\left(t_{(37.1)}=-3.25, p=0.002\right)$, who performed at the expected level $(10.6 \pm 3.7)$ in the Pictures Puzzles task.
After evaluating each participant's performance for this task, we tested whether there was a correlation between participants' scores in the Picture Puzzles task and brain measures that differed between groups. Thus, we conducted the following exploratory analyses: (1) we tested whether there is a correlation between participants' scores in the Picture Puzzles task and properties of early visual areas: the size of visual areas, mean pRF size, and mean pRF eccentricity, and (2) we examined whether there are between-group differences in these relationships.

In TS, performance in the Picture Puzzles task was negatively correlated with mean pRF size in left V2 $(r=-0.56, p=0.017)$ and left V3 $(r=-0.77, p=0.001)$, and trended in the same direction in right $\mathrm{V} 1(r=-0.45, p=0.063)$ and right V3 $(r=$ $-0.46, p=0.056)$. Further, these correlation coefficients were significantly different between TS and controls in left V2 (Fisher's test, $z=2.29, p=0.022)$ and left V3 $(z=3.18, p=0.001)$ (Fig. 5). In TS, performance was also negatively correlated with mean eccentricity in left V3 $(r=-0.53, p=0.023)$ with a similar trend in right $\mathrm{V} 3(r=-0.365, p=0.137)$. However, the difference in the correlation coefficient between TS and controls for left V3 only approached significance (Fisher's test, $z=1.63, p=0.103$ ) (Fig. 5). Finally, within both TS and controls, there were no significant correlations between performance in the Picture Puzzles task and the cortical volume of early visual areas.

These exploratory analyses suggest that mean pRF size and pRF eccentricity of early visual cortex play a role in visuospatial processing in TS but not controls. That is, girls with TS with larger pRFs in early visual areas have lower performance in the visuospatial task than girls with TS with smaller pRFs. Moreover, girls with TS with a larger mean pRF eccentricity in left V3 tend to perform worse in the visuospatial task than controls (with a similar trend in right V3). This negative relationship between performance and eccentricity was not observed in controls.

\section{Discussion}

In this study, we compared properties of early visual areas and their pRFs between girls with TS and controls. Qualitatively, visual field maps V1-V3 and their spatial arrangement across cortex were similar between groups. Quantitatively, we observed three main differences between TS and control girls: (1) early visual areas V1-V3 have smaller volumes in TS compared with controls; this difference is largely drive by smaller anatomical surface area in TS than controls; (2) mean pRF eccentricity is 
lower (closer to the center of gaze) in TS than controls; and (3) there is sparser peripheral VFC in TS compared with controls. We hypothesize that smaller cortical surface area in TS may lead to a lowered number of neurons and consequently result in the sparser peripheral VFC in TS compared with controls (Fig. 4).

Developmental theories of primary visual cortex suggest that this area is fully developed by the age of 5 years. This hypothesis is derived from examination of V1 development in animals, which suggests that it matures early in infancy (Luo and Flanagan, 2007; White and Fitzpatrick, 2007; Huberman et al., 2008) as well as from recent studies finding no differences in $\mathrm{pRF}$ properties of $\mathrm{V} 1-\mathrm{V} 3$ in children (ages 5-12 years) versus adults (22-27 years) (Gomez et al., 2018; Dekker et al., 2019). Given that our youngest participants were 7 years old (Table 1) and early visual cortex is thought to be fully developed by age 5, our results suggest that atypicalities in TS are more likely related to the effect of X-chromosome haploinsufficiency or absence of early hormonal effects of estrogen (Ross et al., 2000) on the brain rather than developmental delays.

Overall, these results indicate that X-chromosome haploinsufficiency might affect functional properties of early visual areas that link to behavior. In addition to $\mathrm{X}$-chromosome haploinsufficiency, abnormalities in properties of early visual areas, as measured by pRF modeling (Dumoulin and Wandell, 2008; Wandell et al., 2015), may stem from the absence of early hormonal effects of estrogen in girls with TS (Ross et al., 2000). The effects of genetic versus gonadal sex on early visual areas can be addressed in future studies using a human analogy of the "four core genotypes" mouse model. If implemented in the context of a longitudinal design, this approach, comparing brains of four genotypes (XX gonadal males and females and XY gonadal males and females), could dissociate between genetic (sex chromosomes) and hormonal (ovarian and testicular secretions) effects (Arnold, 2009).

Our findings demonstrate that functional properties of early visual cortex are affected in TS. Since these early visual areas provide input into higher-order regions (Mishkin et al., 1983), these findings might lead to new perspectives regarding the mechanism through which TS affects other brain regions. V1-V3 provide input into the dorsal visual stream, a pathway ascending from $\mathrm{V} 1$, located in the calcarine sulcus, to posterior parietal cortex. Behavioral and brain imaging studies have reported dorsal visual stream atypicalities in TS. While a large body of research has documented atypicalities in the structure and function of posterior parietal cortex in TS (Lepage et al., 2013; Bray et al.,

A

B
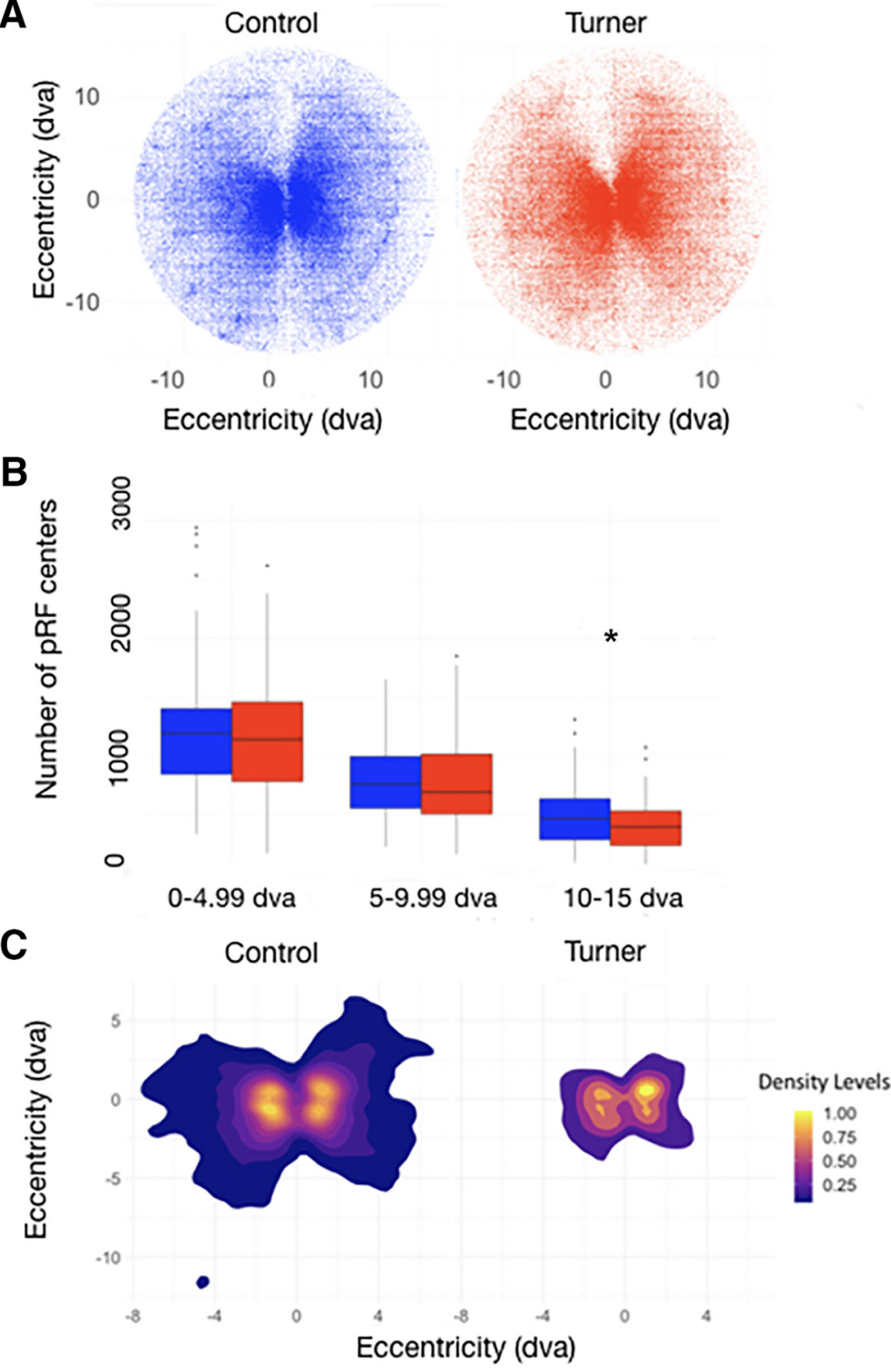

Figure 4. Peripheral pRFs differ between girls with TS and controls. A, Location of pRF centers in the visual field from bilateral V1-V3 is plotted for Turner Syndrome (red) and controls (blue). Each dot represents a voxel. Visual evaluation suggests that the coverage in the peripheral visual field in Turner Syndrome is sparser compared with controls. $\boldsymbol{B}$, Mean number of pRF centers in $0^{\circ}-4.99^{\circ}, 5^{\circ}-9.99^{\circ}$, and $10^{\circ}-15^{\circ}$ bands compared between $\mathrm{TS}$ and controls. ${ }^{*} p<0.05$, significant differences between groups. $C$, kernel density estimation (KDE) of pRF centers' location in the visual field for controls (left) and Turner Syndrome (right).

2013; Green et al., 2014) as well as its functionally connectivity to the frontoparietal attention network (Kesler and Reiss, 2006), accumulating evidence also suggests atypicalities to the cuneus and lingual gyrus, where V2 and V3 reside. For example, neuroimaging studies have shown that, compared with age- and sexmatched controls, both girls and adults with TS show (1) a reduction in volume of the lingual gyrus (Lepage et al., 2013), and the cuneus (Marzelli et al., 2011) and (2) reduced functional connectivity between the cuneus and the whole brain (Xie et al., 2015) as well as reduced functional connectivity within the frontoparietal network (Green et al., 2018). Thus, converging evidence from structural and resting-state MRI shows that TS affects the lingual gyrus and the cuneus as well as the posterior parietal cortex. Given that early visual areas V1-V3 provide inputs to the parietal visual areas of the dorsal stream, our results suggest a new mechanistic explanation for these dorsal stream 

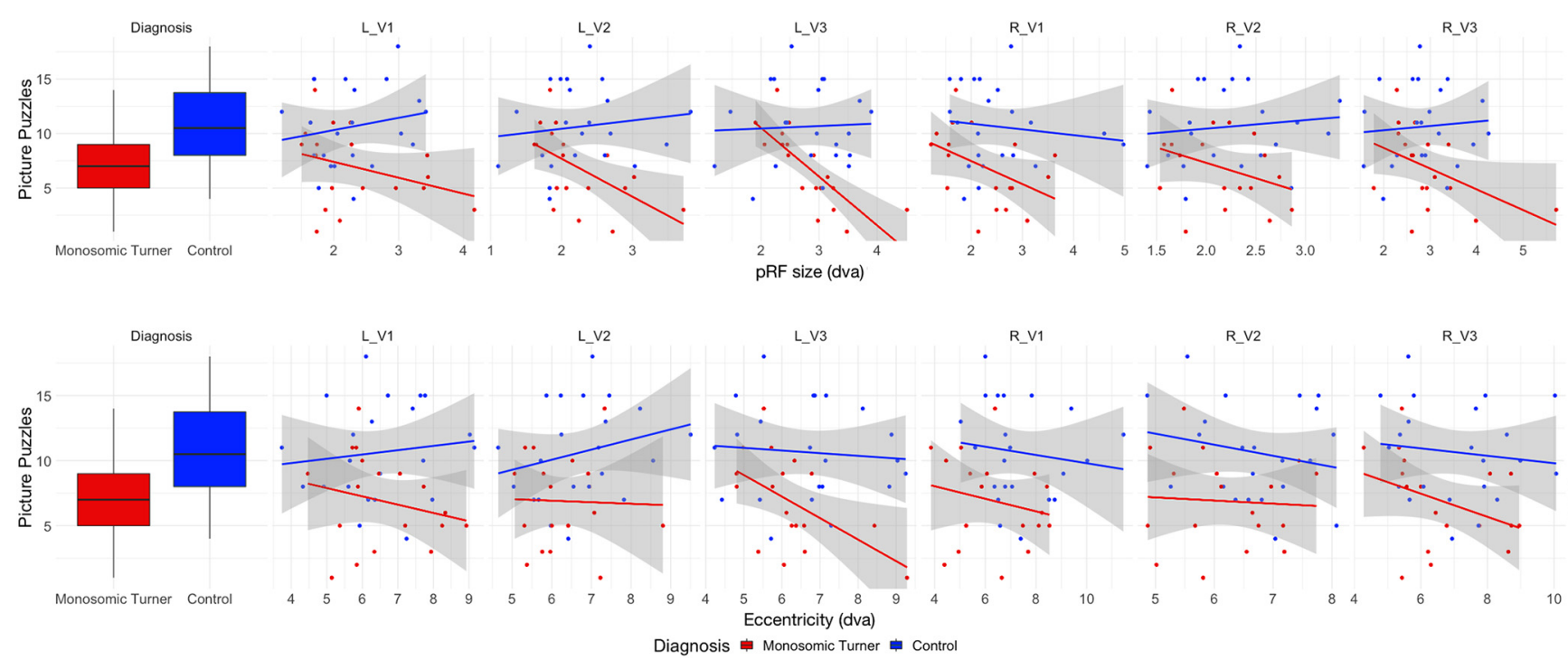

Figure 5. Picture Puzzles performance is negatively correlated with pRF size in Turner Syndrome. Left, Boxplots represent performance in the Picture Puzzles task in 18 Turner Syndrome (red) and 22 control (blue) participants. Right, Pearson correlation quantifying the relationship between participants' scores in the Picture Puzzles task and their pRF size, separately for Turner Syndrome (red) and control (blue) groups. Each dot represents a participant. Shaded area represents $95 \%$ confidence interval (Cl) for each group. Each panel represents a visual area. Top, Correlation between performance and pRF size. Bottom, Correlation between performance and eccentricity.

differences in TS. Specifically, we hypothesize that irregular pRFs in early visual areas may affect pRFs and spatial processing in higher-level visual areas of the dorsal visual stream.

Notably, V1-V3 also provide input into the ventral visual stream (ascending from V1 to ventral temporal cortex), which is involved in visual recognition (Grill-Spector and Malach, 2004). While the potential effect of pRF irregularities in TS in V1-V3 on the ventral stream is currently unknown, neurocognitive evaluation of TS populations suggests that cognitive abilities linked to the ventral stream, specifically those related to poorer recognition of fearful faces (Mazzola et al., 2006) and difficulties in description of scenes (Hepworth and Rovet, 2000), are selectively affected in TS. For example, behavioral studies report poorer configural processing (processing that accounts the relationships between individual components) associated with simplistic or abbreviated description of scenes and events in TS (Reiss et al., 1993; Temple and Carney, 1996; Hepworth and Rovet, 2000). As visual regions involved in processing scenes have a peripheral bias (Levy et al., 2001), one prediction from our data is that reduced peripheral coverage in $\mathrm{V} 1-\mathrm{V} 3$ in TS may affect downstream visual regions involved in scene perception. An alternative possibility, based on empirical evidence showing that visuospatial and executive function deficits are the most commonly and consistently observed impairments in TS (Hong et al., 2009), is that the dorsal stream is more affected than the ventral stream in TS. These hypotheses can be distinguished in future studies of TS that could characterize pRFs and other functional properties in higher-level visual areas in both the dorsal and ventral streams.

While the mean pRF size did not differ between the groups, we found a negative correlation between mean pRF size and visuospatial performance in TS that significantly differed from the brain-behavior correlation in the control group (Fig. 5). We also report a negative correlation between mean pRF eccentricity and visuospatial performance in TS (Fig. 5). However, these correlations did not significantly differ from controls. These exploratory results suggest that girls with TS who have larger pRF size, and their pRFs are placed less centrally (higher eccentricity), perform more poorly at the visuospatial task than those with smaller pRFs and more centralized VFC (lower eccentricity). This finding is consistent with the hypothesis that larger pRFs in early visual areas are associated with poorer spatial processing (de Haas et al., 2014). Thus, this result suggests the possibility that, in the early visual areas of girls with TS, smaller pRFs that are distributed closer to the center of gaze may be developmentally adaptive to the smaller brain surface allocated to early visual cortex. Nevertheless, our data do not exclude the possibility that betweengroup differences in visuospatial characteristic in TS might also be due to differences in spatial processing in higher-level regions in the parietal cortex. Therefore, future research is necessary to build computational models to understand how multilevel hierarchical processing may contribute to the visuospatial deficits in TS. Nonetheless, our findings implicating early visual cortex in visuospatial weakness in TS may serve as a potential target for interventions to improve the development of visuospatial skills known to be affected in this group.

Together, our functional and behavioral data lay fundamental groundwork toward understanding abnormal visual cortical processing in TS. Most significantly, these data suggest, for the first time, that atypicalities in early visual cortex may play a role in the observed visuospatial weakness in TS (Hong et al., 2009; Bray et al., 2013; Green et al., 2014), adding to the rich literature on atypicalities in TS in parietal cortex. Importantly, these findings identify the effect of the X-chromosome haploinsufficiency on basic characteristics of the early visual cortex: (1) smaller surface area, (2) smaller volume, and (3) lesser coverage of the periphery of the visual field $\left(>10^{\circ}\right)$. Overall, these results suggest a paradigm change in the understanding of the effect of sex chromosome dosage variation (i.e., X-monosomy) on the development of visuospatial abilities in humans.

\section{References}

Arnold AP (2009) Mouse models for evaluating sex chromosome effects that cause sex differences in non-gonadal tissues. J Neuroendocrinol 21: $377-386$. 
Bray S, Hoeft F, Hong DS, Reiss AL (2013) Aberrant functional network recruitment of posterior parietal cortex in Turner syndrome. Hum Brain Mapp 34:3117-3128.

Carkeet A, Levi DM, Manny RE (1997) Development of Vernier acuity in childhood. Optom Vis Sci 74:741-750.

Connor CE, Egeth HE, Yantis S (2004) Visual attention: bottom-up versus top-down. Curr Biol 14:R850-R852.

de Haas B, Schwarzkopf DS, Anderson EJ, Rees G (2014) Perceptual load affects spatial tuning of neuronal populations in human early visual cortex. Curr Biol 24:R66-R67.

Dekker TM, Schwarzkopf DS, de Haas B, Nardini M, Sereno MI (2019) Population receptive field tuning properties of visual cortex during childhood. Dev Cogn Neurosci 37:100614.

Dumoulin SO, Wandell BA (2008) Population receptive field estimates in human visual cortex. Neuroimage 39:647-660.

Gomez J, Natu V, Jeska B, Barnett M, Grill-Spector K (2018) Development differentially sculpts receptive fields across early and high-level human visual cortex. Nat Commun 9:788

Green T, Chromik LC, Mazaika PK, Fierro K, Raman MM, Lazzeroni LC, Hong DS, Reiss AL (2014) Aberrant parietal cortex developmental trajectories in girls with Turner syndrome and related visual-spatial cognitive development: a preliminary study. Am J Med Genet B Neuropsychiatr Genet 165B:531-540.

Green T, Saggar M, Ishak A, Hong DS, Reiss AL (2018) X-chromosome effects on attention networks: insights from imaging resting-state networks in Turner syndrome. Cereb Cortex 28:3176-3183.

Grill-Spector K, Malach R (2004) The human visual cortex. Annu Rev Neurosci 27:649-677.

Grill-Spector K, Golarai G, Gabrieli J (2008) Developmental neuroimaging of the human ventral visual cortex. Trends Cogn Sci 12:152-162.

Hepworth SL, Rovet JF (2000) Visual integration difficulties in a 9-year-old girl with Turner syndrome: parallel verbal disabilities? Child Neuropsychol 6:262-273.

Holmes GM (1945) Ferrier lecture: the organization of the visual cortex in man. Proc R Soc Lond B Biol Sci 132:348-361.

Hong D, Scaletta Kent J, Kesler S (2009) Cognitive profile of Turner syndrome. Dev Disabil Res Rev 15:270-278.

Huberman AD, Feller MB, Chapman B (2008) Mechanisms underlying development of visual maps and receptive fields. Annu Rev Neurosci 31:479-509.

Hubel DH, Wiesel TN (1998) Early exploration of the visual cortex. Neuron 20:401-412.

Kay KN, Winawer J, Mezer A, Wandell BA (2013) Compressive spatial summation in human visual cortex. J Neurophysiol 110:481-494.

Kay KN, Weiner KS, Grill-Spector K (2015) Attention reduces spatial uncertainty in human ventral temporal cortex. Curr Biol 25:595-600.

Kesler SR, Reiss AL (2006) Neuro-functional differences associated with arithmetic processing in Turner syndrome. Cereb Cortex 16:849-856.

Korkman M, Kirk U, Kemp S (1998) NESPY: A developmental neuropsychological assessment. San Antonio, TX: The Psychological Corporation.

Lepage JF, Mazaika PK, Hong DS, Raman M, Reiss AL (2013) Cortical brain morphology in young, estrogen-naive, and adolescent, estrogen-treated girls with Turner syndrome. Cereb Cortex 23:2159-2168.
Levy I, Hasson U, Avidan G, Hendler T, Malach R (2001) Center-periphery organization of human object areas. Nat Neurosci 4:533-539.

Luo L, Flanagan JG (2007) Development of continuous and discrete neural maps. Neuron 56:284-300.

Marzelli MJ, Hoeft F, Hong DS, Reiss AL (2011) Neuroanatomical spatial patterns in Turner syndrome. Neuroimage 55:439-447.

Mazzola F, Seigal A, MacAskill A, Corden B, Lawrence K, Skuse DH (2006) Eye tracking and fear recognition deficits in Turner syndrome. Soc Neurosci 1:259-269.

Mishkin M, Ungerleider LG, Macko KA (1983) Object vision and spatial vision: two cortical pathways. Trends Neurosci 6:414-417.

Raznahan A, Cutter W, Lalonde F, Robertson D, Daly E, Conway GS, Skuse DH, Ross J, Lerch JP, Giedd JN, Murphy DD (2010) Cortical anatomy in human X monosomy. Neuroimage 49:2915-2923.

Reiss AL, Freund L, Plotnick L, Baumgardner T, Green K, Sozer AC, Reader M, Boehm C, Denckla MB (1993) The effects of X monosomy on brain development: monozygotic twins discorcant for Turner's syndrome. Ann Neurol 34:95-107.

Ross JL, Roeltgen D, Feuillan P, Kushner H, Cutler GB Jr (2000) Use of estrogen in young girls with Turner syndrome: effects on memory. Neurology 54:164-170

Sereno MI, Dale AM, Reppas JB, Kwong KK, Belliveau JW, Brady TJ, Rosen BR, Tootell RB (1995) Borders of multiple visual areas in humans revealed by functional magnetic resonance imaging. Science 268:889-893.

Skoczenski AM, Norcia AM (2016) Late Maturation of Visual Hyperacuity. Psychological Science 13:537-541.

Stochholm K, Juul S, Juel K, Naeraa RW, Gravholt CH (2006) Prevalence, incidence, diagnostic delay, and mortality in Turner syndrome. J Clin Endocrinol Metab 91:3897-3902.

Temple CM, Carney R (1996) Reading skills in children with Turner's syndrome: an analysis of hyperplexia. Cortex 32:335-345.

Wandell BA, Winawer J (2011) Imaging retinotopic maps in the human brain. Vision Res 51:718-737.

Wandell BA, Winawer J (2015) Computational neuroimaging and population receptive fields. Trends Cogn Sci 19:349-357.

Wandell BA, Dumoulin SO, Brewer AA (2007) Visual field maps in human cortex. Neuron 56:366-383.

Wandell BA, Winawer J, Kay KN (2015) Computational modeling of responses in human visual cortex. In: Brain mapping, pp 651-659. Amsterdam: Elsevier.

Wechsler D (2003) Wechsler intelligence scale for children, Ed 4. London: Pearson.

White LE, Fitzpatrick D (2007) Vision and cortical map development. Neuron 56:327-338.

Witthoft N, Nguyen ML, Golarai G, LaRocque KF, Liberman A, Smith ME, Grill-Spector K (2014) Where is human V4? Predicting the location of hV4 and VO1 from cortical folding. Cereb Cortex 24:2401-2408.

Xie S, Zhang Z, Zhao Q, Zhang J, Zhong S, Bi Y, He Y, Pan H, Gong G (2015) The effects of $\mathrm{X}$ chromosome loss on neuroanatomical and cognitive phenotypes during adolescence: a multi-modal structural MRI and diffusion tensor imaging study. Cereb Cortex 25:2842-2853. 